\title{
Interacting Modified Variable Chaplygin Gas in a Non-flat Universe
}

\author{
Mubasher Jamil* and Muneer Ahmad Rashid ${ }^{\dagger}$ \\ Center for Advanced Mathematics and Physics \\ National University of Sciences and Technology \\ Peshawar Road, Rawalpindi - 46000, Pakistan
}

November 10, 2018

\begin{abstract}
A unified model of dark energy and matter is presented using the modified variable Chaplygin gas for interacting dark energy in a non-flat universe. The two entities interact with each other non-gravitationally which involves a coupling constant. Due to dynamic interaction, the variation in this constant arises that henceforth changes the equations of state of these quantities. We have derived the effective equations of state corresponding to matter and dark energy in this interacting model. Moreover, the case of phantom energy is deduced by putting constraints on the parameters involved.
\end{abstract}

Keywords: Interaction; Chaplygin Gas; Dark Matter; Dark energy; Coupling constant.

\section{Introduction}

Hosts of results coming from the observations of WMAP [1, 2] have convincingly shown the validity of standard Big Bang model with cosmological constant $\Lambda$, so called $\Lambda$ CDM model. Surprisingly the energy density corresponding to $\Lambda$ is two third of the critical density or $\Omega_{\Lambda} \approx 0.7$, the remaining part is due to baryonic and non-baryonic dark matter. The genesis of this energy, commonly called the dark energy, and its subsequent evolution poses serious problems to investigate. The dark energy is commonly represented by a barotropic equation of state (EoS) $p=-\rho$ (or $\omega=-1$ ). Numerous models have been proposed in the literature to explain the cosmic

${ }^{*}$ Corresponding author: mjamil@camp.edu.pk

${ }^{\dagger}$ muneerrshd@yahoo.com 
accelerated expansion which include those based on homogeneous and time dependent scalar field termed as quintessence [3], phantom energy having EoS $\omega<-1$ [4], Chaplygin gas (CG) with the EoS $p=-X / \rho$ [5], dissipative or viscous cosmology [6] and modified gravity models including Dvali-Gabadadze-Porrati (DGP) model [7] and higher order $f(R)$ gravity theories [8].

Among all of these alternative theories, the Chaplygin gas effectively explains the evolution of the universe right from the earlier matter dominated era (decelerating phase) to the late dark energy dominated era (accelerating phase). This CG was first introduced in the aerodynamical context [9] and has been largely investigated in recent years due to its adequate efficiency in interpolating the astrophysical data.

The energy conservation equation $\dot{\rho}+3 H(\rho+p)=0$ yields

$$
\rho=\sqrt{X+\frac{Y}{a^{6}}},
$$

where $X$ is a positive constant and $Y$ is the constant of integration. Above, $p$ and $\rho$ are the pressure and energy densities of the CG. Thus small values of $a(t)$ mimic dust phase while large values represent dark energy phase of the universe. The CG model favors a spatially flat universe with $95.4 \%$ confidence level which agrees with the observational data of Sloan Digital Sky Survey (SDSS) and Supernova Legacy Survey (SNLS) [10]. It also possesses a property of giving accelerated expansion even if it gets coupled with other scalar fields like quintessence or dissipative matter fields [11]. Due to its this effectiveness, various generalizations of CG have been proposed in the literature (see e.g. [12, 13, 14, 15, 16, 17, 18, 19, 20, 21]). All these models based on the inhomogeneous Chaplygin gas offer unified picture of dark matter and dark energy (sometimes called dark matter-dark energy unification) [22]. But at the same time CG has a drawback as it produces oscillations or exponential blow up of dark matter power spectrum, which is inconsistent with the observations [23]. Similar results have been obtained in later generalizations of CG [24].

One of the long standing problems in cosmology for the past decade is the so-called "cosmic coincidence problem" which naively asks: If the universe evolves from the earlier quintessence $(\omega>-1)$ to the later phantom regime $(\omega<-1)$, then why $\omega=-1$ crossing is occurring at the present time. The astrophysical data seems to favor the evolving dark energy in which the EoS parameter $\omega$ changes at different cosmic epochs [25]. This problem can be rephrased alternatively as: In any cosmological model explaining dark energy, the ratio of matter to dark energy density $r_{m}$ is expected to decrease with the expansion of the universe. However the observations suggest that this ratio is of the order unity. It may implies the transfer of energy between these two entities to keep a delicate balance at the current time. The models in which this interaction is investigated are called the 'interacting dark energy models'. The interaction involves a coupling constant that determines the strength of this interaction.

Earlier, it had been suggested that interacting models of CG with dark matter can effectively ameliorate the coincidence problem which is not possible in pure CG models [26, 27, 28]. More- 
over, this problem has also been investigated in the context of dissipative CG and successfully explains the cosmic conundrum problem [11]. The interacting model effectively explains the phantom divide $\omega=-1$ i.e. the transition from $\omega>-1$ to $\omega<-1$ [25, 29, 30]. It also rules out the possibility of the exotic phenomenon termed 'Big Rip' which is the infinite expansion of the universe in a finite time [31]. It also yields stable stationary attractor solution of the Friedmann-Robertson-Walker (FRW) equations at late times. So if dark energy evolves then it would be worthwhile to find its effective EoS. This motivates us to investigate the more generalized form of interacting CG and to find the effective EoS of dark energy and matter in the respective model. The formalism adopted here is from Ref. [19].

\section{Interacting modified variable Chaplygin gas}

We assume the background spacetime to be spatially homogeneous and isotropic represented by the FRW metric

$$
d s^{2}=-d t^{2}+a^{2}(t)\left[\frac{d r^{2}}{1-k r^{2}}+r^{2}\left(d \theta^{2}+\sin ^{2} \theta d \phi^{2}\right)\right] .
$$

Here $a(t)$ is the scale factor and $k=-1,0,+1$ represent spatially hyperbolic, flat and closed universes respectively. The corresponding Einstein field equation is

$$
H^{2}+\frac{k}{a^{2}}=\frac{1}{3 M_{p}^{2}}\left(\rho_{\Lambda}+\rho_{m}\right)
$$

where $M_{p}^{2}=(8 \pi G)^{-1}$ is the modified Planck mass. Moreover, the energy conservation for our dynamical system is given by

$$
\dot{\rho}_{\Lambda}+\dot{\rho}_{m}+3 H\left[\left(1+\omega_{\Lambda}\right) \rho_{\Lambda}+\rho_{m}\right]=0
$$

where we have taken the EoS for the dark energy $p_{\Lambda}=\omega_{\Lambda} \rho_{\Lambda}$ and for the matter part $p_{m}=0$ $\left(\right.$ or $\left.\omega_{m}=0\right)$.

To derive the general form of the interaction between $\mathrm{CG}$ and matter, we start by considering the action of the scalar-tensor theory of gravitation given by

$$
S_{S T}=\int d^{4} x \sqrt{-g}\left[\frac{\Re}{2}-\frac{1}{2} \partial_{\mu} \phi \partial^{\mu} \phi+\frac{1}{\Theta^{2}(\phi)} L_{m}\left(\xi, \partial \xi, \Theta^{-1} g_{\mu \nu}\right)\right],
$$

where $\Re$ is the Ricci scalar, $\phi$ is the spatially homogeneous scalar field, $L_{m}$ represents the matter Lagrangian, $\xi$ is the collective degrees of freedom of matter and $\Theta^{-2}$ is the coupling function between matter and the scalar field. Using the action $S_{S T}$, the interaction term $Q$ can be written as 26,32

$$
Q=\rho_{m} H \frac{d \ln \Theta^{\prime}}{d \ln a},
$$


where $\Theta^{\prime} \equiv \Theta(a)^{\left(3 \omega_{m}-1\right) / 2}$. Letting

$$
\Theta^{\prime}(a)=\Theta_{o} e^{3 \int b^{2}\left(\frac{\rho_{m}+\rho_{\Lambda}}{\rho_{m}}\right) d \ln a} .
$$

Substitution of Eq. (7) in (6) yields

$$
Q=3 H b^{2}\left(\rho_{\Lambda}+\rho_{m}\right)
$$

which is the general form of the interaction between CG and matter. Here $b^{2}$ is the dimensionless coupling constant (also called 'transfer strength') for the interaction. The positive $b^{2}$ is responsible for the transition from CG to matter and vice versa for negative $b^{2}$. Sometimes this constant is taken in the range $[0,1]$ (see Ref. [26]). Note that if $b^{2}=0$ then it represents the non-interacting FRW model while $b^{2}=1$ yields complete transfer of energy from CG to matter. Recently, it is reported that this interaction is observed in the Abell cluster A586 showing a transition of dark energy into dark matter and vice versa [33]. Therefore the theoretical interacting models are phenomenologically consistent with the observations.

Let us assume an interaction $Q=\Gamma \rho_{\Lambda}$ between $\mathrm{CG}$ and dark matter, where $\Gamma$ is decay rate. Then Eq. (4) can be divided into two parts corresponding to $\rho_{\Lambda}$ and $\rho_{m}$ as

$$
\begin{aligned}
\dot{\rho}_{\Lambda}+3 H\left(1+\omega_{\Lambda}\right) \rho_{\Lambda} & =-Q, \\
\dot{\rho}_{m}+3 H \rho_{m} & =Q,
\end{aligned}
$$

respectively. Eqs. (9) and (10) show that the energy conservation for dark energy and matter would not hold independently if there is an interaction between them but hold for the total interacting system as manifested through Eq. (4). Further, we define the density ratio $r_{m}$ as $r_{m} \equiv \frac{\rho_{m}}{\rho_{\Lambda}}$. To study how this density ratio evolves with time, we differentiate $r_{m}$ with respect to $t$ and obtain

$$
\dot{r}_{m}=\frac{d r_{m}}{d t}=\frac{\rho_{m}}{\rho_{\Lambda}}\left[\frac{\dot{\rho}_{m}}{\rho_{m}}-\frac{\dot{\rho}_{\Lambda}}{\rho_{\Lambda}}\right],
$$

Using Eqs. (9) and (10) in (11), we get

$$
\dot{r}_{m}=3 H r_{m}\left[\omega_{\Lambda}+\frac{1+r_{m}}{r_{m}} \frac{\Gamma}{3 H}\right] .
$$

Defining the effective EoS for dark energy and matter as [34]

$$
\omega_{\Lambda}^{e f f}=\omega_{\Lambda}+\frac{\Gamma}{3 H}, \quad \omega_{m}^{e f f}=\frac{-1}{r_{m}} \frac{\Gamma}{3 H},
$$

which involve the contribution from the interaction between matter and dark energy. Using Eq. (13) in Eqs. (9) and (10), we get

$$
\begin{aligned}
\dot{\rho}_{\Lambda}+3 H\left(1+\omega_{\Lambda}^{e f f}\right) \rho_{\Lambda} & =0 \\
\dot{\rho}_{m}+3 H\left(1+\omega_{m}^{e f f}\right) \rho_{m} & =0 .
\end{aligned}
$$


From the standard FRW model, the density parameters corresponding to matter, dark energy and curvature are defined as

$$
\begin{aligned}
\Omega_{m} & =\frac{\rho_{m}}{\rho_{c r}}=\frac{\rho_{m}}{3 H^{2} M_{p}^{2}}, \\
\Omega_{\Lambda} & =\frac{\rho_{\Lambda}}{\rho_{c r}}=\frac{\rho_{\Lambda}}{3 H^{2} M_{p}^{2}}, \\
\Omega_{k} & =\frac{k}{a^{2} H^{2}} .
\end{aligned}
$$

The parameter $\Omega_{k}$ represents the contribution in the total energy density from the spatial curvature and is constrained as $-0.0175<\Omega_{k}<0.0085$ with $95 \%$ confidence level by current observations [35]. It is shown that a non-zero positive curvature parameter $k$ allows for a bounce, thereby preventing the cosmic singularities without violating the null energy condition $\rho+p \geq 0$ 36].

The density parameters in Eqs. (16), (17) and (18) are related as

$$
\Omega_{m}+\Omega_{\Lambda}=1+\Omega_{k} .
$$

Using definition of $r_{m}$ and Eq. (19), we can write

$$
r_{m}=\frac{1+\Omega_{k}-\Omega_{\Lambda}}{\Omega_{\Lambda}} .
$$

In our generalized model, we choose the modified variable Chaplygin gas (MVG) given by

$$
p_{\Lambda}=A \rho_{\Lambda}-\frac{B(a)}{\rho_{\Lambda}^{\alpha}}, B(a)=B_{o} a^{-n} .
$$

Here $A, B_{o}$ and $n$ are positive constants and $0 \leq \alpha \leq 1$. Notice that for $B=0$, Eq. (21) represents the barotropic EoS (or barotropic fluid). In general, the barotropic EoS $p=A \rho$ represents various types of mediums. For instance, $A=-1$ gives cosmological constant or de Sitter vacuum; for $A=-2 / 3$, it corresponds to domain walls; if $A=-1 / 3$ it gives cosmic strings; while $A=0$ corresponds to dust or matter; $A=1 / 3$ yields the EoS for relativistic gas like photon radiation; $A=2 / 3$ gives the perfect gas and $A=1$ represents the ultra-stiff matter 37. If $B(a)=B_{o}$ in Eq. (21), it gives EoS of modified CG. Again if $A=0$ and $\alpha=1$, it represents the usual CG. Recently it is deduced using the latest supernovae data that models with $\alpha>1$ are also possible [38]. Notice that for $A=0$, Eq. (21) yields a fluid with negative pressure. Such a fluid is generally characterized in the quintessence regime.

The modified form of the CG is also phenomenologically motivated and can explain the flat rotational curves of galaxies [39]. The galactic rotational velocity $V_{c}$ is related with the MVG parameter $A$ as $V_{c}=\sqrt{2 A}$ and the density parameter $\rho$ is related to the radial size of the galaxy as $\rho=\frac{A}{2 \pi G r^{2}}$. At high densities, the first term in MVG dominates and gives the flat rotational curve that is consistent with with observations for galaxies. The parameter $A$ varies from galaxy to galaxy due to variations in $V_{c}$. 
The density evolution of MVG is given by

$$
\rho_{\Lambda}=\left(\Delta a^{-n}+C a^{-s}\right)^{\frac{1}{1+\alpha}},
$$

where $s \equiv 3(1+\alpha)(1+A), \Delta \equiv \frac{3(1+\alpha) B_{o}}{s-n}$ and $C$ is the constant of integration. Differentiating Eq. (22) we get

$$
\dot{\rho}_{\Lambda}=\frac{-H}{1+\alpha}\left[\left(\Delta a^{-n}+C a^{-s}\right)^{\frac{-\alpha}{1+\alpha}}\left(\Delta n a^{-n}+C s a^{-s}\right)\right]
$$

Further, choosing the decay rate to be [40]

$$
\Gamma=3 b^{2} H\left(1+r_{m}\right) .
$$

Substituting Eq. (24) in Eq. (9) and using $Q=3 b^{2} H\left(1+r_{m}\right) \rho_{\Lambda}$, we obtain

$$
\omega_{\Lambda}=\frac{1}{3(1+\alpha)}\left[\left(\Delta a^{-n}+C a^{-s}\right)^{-1}\left(\Delta n a^{-n}+C s a^{-s}\right)\right]-b^{2} \frac{1+\Omega_{k}}{\Omega_{\Lambda}}-1 .
$$

Using Eq. (20) and (24), we have

$$
\Gamma=3 b^{2} H \frac{1+\Omega_{k}}{\Omega_{\Lambda}}
$$

Thus using Eqs. (14), (25) and (26), we arrive at the effective EoS for dark energy as

$$
\omega_{\Lambda}^{e f f}=\frac{1}{3(1+\alpha)}\left[\left(\Delta a^{-n}+C a^{-s}\right)^{-1}\left(\Delta n a^{-n}+C s a^{-s}\right)\right]-1 .
$$

Notice that the cosmological models with phantom energy arise when $\omega_{\Lambda}^{\text {eff }}<-1$ which is possible if the quantity in the square brackets in Eq. (27) is less then zero. Therefore, we have two possible cases:

Case (1). If $\left(\Delta a^{-n}+C a^{-s}\right)>0$ and $\left(\Delta n a^{-n}+C s a^{-s}\right)<0$, then we have a constraint on $a(t)$

$$
\frac{-C}{\Delta}<a^{s-n}<\frac{-C s}{\Delta n} \text {. }
$$

This is possible when $\frac{-C}{\Delta}>0$ and $s>n$.

Case (2). If $\left(\Delta a^{-n}+C a^{-s}\right)<0$ and $\left(\Delta n a^{-n}+C s a^{-s}\right)>0$, then we have

$$
\frac{-C s}{\Delta n}<a^{s-n}<\frac{-C}{\Delta}
$$

here $a(t)$ will be restricted when $\frac{-C}{\Delta}>0$ and $s<n$.

Thus the phantom dark energy case is possible if scale factor $a(t)$ is constrained as given in Eqs. (28) and (29). Also, Eq. (27) represents usual cosmological constant if the quantity in the square brackets vanishes identically.

Also we note that the quantity in Eq. (22) must be positive so that $a(t)>\left(\frac{-C}{\Delta}\right)^{\frac{1}{s-n}}$. Therefore, the minimum value of $a(t)$ lies at

$$
a_{\text {min }}=\left(\frac{-C}{\Delta}\right)^{\frac{1}{s-n}}
$$


Therefore the universe can experience a bounce before reaching the singularity when $a>a_{\min }$ which corresponds to the bouncing universe model. This bounce is possible due to our assumption of positive curvature (see Ref. [41] for a review on bouncing cosmologies). Moreover, the effective EoS of matter is determined from Eqs. (13), (20) and (24) as

$$
\omega_{m}^{e f f}=-b^{2}\left(1+\frac{\Omega_{\Lambda}}{\Omega_{m}}\right)
$$

Using $\Omega_{\Lambda}=0.7$ and $\Omega_{m}=0.3$, we find from Eq. (31)

$$
\omega_{m}^{e f f}=-3.33 b^{2}
$$

Note that $\omega_{m}^{e f f}$ depends only on the coupling constant. If there is no interaction then $\omega_{m}^{e f f}=0$. In particular if $0 \leq b^{2} \leq 1$, it implies $-3.33 \leq \omega_{m}^{\text {eff }} \leq 0$. Therefore in the interacting model, matter can also behave as a fluid with negative pressure which can cause the cosmic acceleration.

\section{Conclusion}

In summary, we have determined the effective equations of state for matter and dark energy in the generalized model of interacting Chaplygin gas. In particular for dark energy, the modified variable Chaplygin gas equation of state is used. Since dark energy interacts with matter then due to energy exchange, the EoS of the both the quantities will be modified. The effective EoS of Chaplygin gas $\omega_{\Lambda}^{e f f}$ also describes the EoS of phantom energy if the scale factor is constrained by either Eq. (28) or (29). As discussed, the interaction is observed in the near galactic cluster Abell A586, we may hope to determine the effective EoS of both matter and dark energy as well through future astrophysical observations that will finally put further constraints on the parameters in the model. Also it is shown that a universe filled with the Chaplygin gas can bounce from the singularity if $a(t)$ is greater then $a_{m i n}$, where the later describes the minimum value of scale factor at the bounce. Finally, it is shown that matter can also generate negative pressure along with dark energy to cause cosmic expansion.

\section{Acknowledgment}

We would like to thank anonymous referees for giving useful comments to improve this work.

\section{References}

[1] Spergel D N et al, 2003 Ap. J. Supp. 148175

[2] Wang B et al, 2007 Nuc. Phys. B $\mathbf{7 7 8} 69$ 
[3] Wang L et al, 2000 Ap. J. 53017

[4] Caldwell R R, 2002 Phys. Lett. B 54523

[5] Kamenshchik A et al, 2001 Phys. Lett. B 511265

[6] Ren J and Meng X, 2006 Phys. Lett. B 6331

[7] Dvali G R et al, 2000 Phys. Lett. B $\mathbf{4 8 4} 112$

[8] Nojiri S and Odintsov S D, 2004 Phys. Lett. B 599137

[9] Chaplygin S, 1904 Sci. Mem. Moscow Univ. Math. Phys. 211

[10] Xen W P and Wei Y H, 2007 Chin. Phys. Lett. 24843

[11] Chimento L P et al 2006 Phys. Rev. D 67103007

[12] Bento M C et al, 2002 Phys. Rev. D 66043507

[13] Cruz N et al, 2007 Phys. Lett. B 646177

[14] Benaoum H B, hep-th/0205140

[15] Setare M R, 2007 Phys. Lett. B 648329

[16] Setare M R, 2007 Phys. Lett. B 6541

[17] Setare M R, hep-th/0712.4004

[18] Zhang H and Zhu Z, arXiv:0704.3121

[19] Setare M R, 2007 Eur. Phys. J. C 52689

[20] Guo Z and Zhang Y, 2007 Phys. Lett. B 645326

[21] Debnath U, 2007 Ap. Sp. Sci. 312295

[22] Belin et al, 2002 Phys. Lett. B 53517

[23] Sandvik H B et al, 2004 Phys. Rev. D 69123524

[24] Carturan D and Finelli F, 2003 Phys. Rev. D 68103501

[25] Sadjadi H M and Alimohammadi M, 2006 Phys. Rev. D 74103007

[26] Zhang H and Zhu Z, 2006 Phys. Rev. D 73043518

[27] Wu P and Yu H, 2007 Class. Quantum Grav. 244661

[28] del Campo S et al, 2006 Phys. Rev. D 74023501 
[29] Setare M R, 2006 Phys. Lett. B 6421

[30] Setare M R, 2007 Eur. Phys. J. C 50991

[31] Wu Y B et al, 2007 Gen. Relativ. Gravit. 39653

[32] Curbelo R et al, 2006 Class. Quant. Grav. 231585

[33] Bertolami O et al, 2007 Phys. Lett. B 654165

[34] Kim H et al, 2006 Phys. Lett. B 632605

[35] Waterhouse T P and Zipin J P, astro-ph/0804.1771

[36] Paris C M and Visser M, 1999 Phys. Lett. B 45590

[37] Dymnikova I and Fil'chenkov M, 2002 Phys. Lett. B 545214

[38] Bertolami O et al, 2004 Mon. Not. Roy. Ast. Soc. 353329

[39] Tekola A G, gr-qc/0706.0804

[40] Wang B et al, 2005 Phys. Lett. B 624141

[41] Novello M and Perez-Bergliaffa S E, astro-ph/0802.1634 